\title{
Similarity Quotients as Final Coalgebras
}

\author{
Paul Blain Levy \\ University of Birmingham, UK \\ P.B.Levy@cs.bham.ac.uk
}

\begin{abstract}
We give a general framework connecing a branching time relation on nodes of a transition system to a final coalgebra for a suitable endofunctor. Examples of relations treated by our theory include bisimilarity, similarity, upper and lower similarity for transition systems with divergence, similarity for discrete probabilistic systems, and nested similarity. Our results describe firstly how to characterize the relation in terms of a given final coalgebra, and secondly how to construct a final coalgebra using the relation.

Our theory uses a notion of "relator" based on earlier work of Thijs. But whereas a relator must preserve binary composition in Thijs' framework, it only laxly preserves composition in ours. It is this weaker requirement that allows nested similarity to be an example.
\end{abstract}

\section{Introduction}

A series of influential papers including 1111/17/8,19] have developed a coalgebraic account of bisimulation, based on the following principles.

- A transition system may be regarded as a coalgebra for a suitable endofunctor $F$ on Set (or another category).

- Bisimulation can be defined in terms of an operation on relations, called a "relational extension" or "relator".

- This operation may be obtained directly from $F$, if $F$ preserves quasipullbacks 3 .

- Given a final $F$-coalgebra, two nodes of transition systems are bisimilar iff they have the same anamorphic image-i.e. image in the final coalgebra.

- Any coalgebra can be quotiented by bisimilarity to give an extensional coalgebra - one in which bisimilarity is just equality.

- One may construct a final coalgebra by taking the extensional quotient of a sufficiently large coalgebra.

Thus a final $F$-coalgebra provides a "universe of processes" according to the viewpoint that bisimilarity is the appropriate semantic equivalence.

More recently 247/21322 there have been several coalgebraic studies of simulation, in which the final $F$-coalgebra carries a preorder. This is valuable for someone who wants to study bisimilarity and similarity together: equality

^ Supported by EPSRC Advanced Research Fellowship EP/E056091.

M. Hofmann (Ed.): FOSSACS 2011, LNCS 6604, pp. 27-41, 2011.

(C) Springer-Verlag Berlin Heidelberg 2011 
represents bisimilarity, and the preorder represents similarity. But someone who is exclusively interested in similarity will want the universe of processes to be a poset: if two nodes are mutually similar, they should be equal. In this paper we shall see that such a universe is also a final coalgebra, for a suitable endofunctor $H$ on the category of posets.

For example, consider countably branching transition systems. In this case, we shall see that $H$ maps a poset $A$ to the set of countably generated lower sets, ordered by inclusion. A final $H$-coalgebra is a universe for similarity, in two senses.

- On the one hand, we can use a final $H$-coalgebra to characterize similarity, by regarding a transition system as a discretely ordered $H$-coalgebra.

- On the other hand, we can construct a final $H$-coalgebra, by taking a sufficiently large transition system and quotienting by similarity.

We give this theory in Sect. 4. But first, in Sect. 3. we introduce the notion of relator, which gives many notions of simulation, e.g. for transition systems with divergence and Markov chains. Finally, in Sect. 5] we look at the example of 2-nested simulation; this requires a generalization of our theory where relations are replaced by indexed families of relations.

\section{Mathematical Preliminaries}

\section{Definition 1. (Relations)}

1. For sets $X$ and $Y$, we write $X \stackrel{\mathcal{R}}{\longrightarrow} Y$ when $\mathcal{R}$ is a relation from $X$ to $Y$, and $\operatorname{Rel}(X, Y)$ for the complete lattice of relations ordered by inclusion.

2. $X \stackrel{(=X)}{\longrightarrow} X$ is the equality relation on $X$.

3. Given relations $X \stackrel{\mathcal{R}}{\longrightarrow} Y \stackrel{\mathcal{S}}{\longrightarrow} Z$, we write $X \stackrel{\mathcal{R} ; \mathcal{S}}{\longrightarrow} Z$ for the composite.

4. Given functions $Z \stackrel{f}{\longrightarrow} X$ and $W \stackrel{g}{\longrightarrow} Y$, and a relation $X \stackrel{\mathcal{R}}{\longrightarrow} Y$, we write $Z \stackrel{(f, g)^{-1} \mathcal{R}}{\longrightarrow} W$ for the inverse image $\{(z, w) \in Z \times W \mid f(z) \mathcal{R} g(w)\}$.

5. Given a relation $X \stackrel{\mathcal{R}}{\longrightarrow} Y$, we write $Y \stackrel{\mathcal{R}^{c}}{\longrightarrow} X$ for its converse. $\mathcal{R}$ is difunctional when $\mathcal{R} ; \mathcal{R}^{\mathrm{c}} ; \mathcal{R} \subseteq \mathcal{R}$.

Definition 2. (Preordered sets)

1. A preordered set $A$ is a set $A_{0}$ with a preorder $\leqslant_{A}$. It is a poset (setoid, discrete setoid) when $\leqslant_{A}$ is a partial order (an equivalence relation, the equality relation).

2. We write Preord (Poset, Setoid, DiscSetoid) for the category of preordered sets (posets, setoids, discrete setoids) and monotone functions.

3. The functor $\Delta:$ Set $\longrightarrow$ Preord maps $X$ to $\left(X,=_{X}\right)$ and $X \stackrel{f}{\longrightarrow} Y$ to $f$. This gives an isomorphism Set $\cong$ DiscSetoid. 
4. Let $A$ and $B$ be preordered sets. $A$ bimodule $A \stackrel{\mathcal{R}}{\longrightarrow} B$ is a relation such that $\left(\leqslant_{A}\right) ; \mathcal{R} ;\left(\leqslant_{B}\right) \subseteq \mathcal{R}$. We write $\operatorname{Bimod}(A, B)$ for the complete lattice of bimodules, ordered by inclusion. For an arbitrary relation $A_{0} \stackrel{\mathcal{R}}{\longrightarrow} B_{0}$, its bimodule closure $A \stackrel{\overline{\mathcal{R}}}{\longrightarrow} B$ is $\left(\leqslant_{A}\right) ; \mathcal{R} ;\left(\leqslant_{B}\right)$.

Definition 3. (Quotienting)

1. Let $A$ be a preordered set. For $x \in A$, its principal lower set $[x]_{A}$ is $\left\{y \in A \mid y \leqslant_{A} x\right\}$. The quotient poset $Q A$ is $\left\{[x]_{A} \mid x \in A\right\}$ ordered by inclusion. (This is isomorphic to the quotient of $A$ by the equivalence relation $\left(\leqslant_{A}\right) \cap\left(\geqslant_{A}\right)$.) We write $A \stackrel{p_{A}}{\longrightarrow} Q A$ for the function $x \mapsto[x]_{A}$.

2. Let $A$ and $B$ be preordered sets and $A \stackrel{f}{\longrightarrow} B$ a monotone function. The monotone function $Q A \stackrel{Q f}{\longrightarrow} Q B$ maps $[x]_{A} \mapsto[f(x)]_{B}$.

3. Let $A$ and $B$ be preordered sets and $A \stackrel{\mathcal{R}}{\longrightarrow} B$ a bimodule. The bimodule $Q A \stackrel{Q \mathcal{R}}{\longrightarrow} Q B$ relates $[x]_{A}$ to $[y]_{B}$ iff $x \mathcal{R} y$.

We give some examples of endofunctors on Set.

Definition 4. 1. For any set $X$ and class $K$ of cardinals, we write $\mathcal{P}^{K} X$ for the set of subsets $X$ with cardinality in $K . \mathcal{P}$ is the endofunctor on Set mapping $X$ to the set of subsets of $X$ and $X \stackrel{f}{\longrightarrow} Y$ to $u \mapsto\{f(x) \mid x \in u\}$. It has subfunctors $\mathcal{P}^{[0, \kappa)}$ and $\mathcal{P}^{[1, \kappa)}$ where $\kappa$ is a cardinal or $\infty$.

2. Maybe is the endofunctor on Set mapping $X$ to $X+1=\{$ Just $x \mid x \in X\} \cup$ $\{\Uparrow\}$ and $X \stackrel{f}{\longrightarrow} Y$ to Just $x \mapsto$ Just $f(x), \Uparrow \mapsto \Uparrow$.

3. $A$ discrete subprobability distribution on a set $X$ is a function $d: X \longrightarrow$ $[0,1]$ such that $\sum_{x \in X} d_{x} \leqslant 1$ (so $d$ is countably supported). For any $U \subseteq X$ we write $d U \stackrel{\text { def }}{=} \sum_{x \in U} d_{x}$, and we write $d \Uparrow \stackrel{\text { def }}{=} 1-d(X)$. D is the endofunctor on Set mapping $X$ to the set of discrete subprobability distributions on $X$ and $X \stackrel{f}{\longrightarrow} Y \quad$ to $d \mapsto\left(y \mapsto d\left(f^{-1}\{y\}\right)\right)$.

Definition 5. Let $\mathcal{C}$ be a category.

1. Let $F$ be an endofunctor on $\mathcal{C}$. An $F$-coalgebra $M$ is a $\mathcal{C}$-object $M$ and morphism $M \cdot \stackrel{\zeta_{M}}{\longrightarrow} F M \cdot$. We write $\operatorname{Coalg}(\mathcal{C}, F)$ for the category of $F$-coalgebras and homomorphisms.

2. Let $F$ and $G$ be endofunctors on $\mathcal{C}$, and $F \stackrel{\alpha}{\longrightarrow} G$ a natural transformation. We write $\operatorname{Coalg}(\mathcal{C}, \alpha): \operatorname{Coalg}(\mathcal{C}, F) \longrightarrow \operatorname{Coalg}(\mathcal{C}, G)$ for the functor mapping $M$ to $\left(M \cdot, \zeta_{M} ; \alpha_{M} \cdot\right)$ and $M \stackrel{f}{\longrightarrow} N$ to $f$. 
Examples of coalgebras:

- a transition system is a $\mathcal{P}$-coalgebra

- a countably branching transition system is a $\mathcal{P}^{\left[0, \aleph_{0}\right]_{\text {-coalgebra }}}$

- a transition system with divergence is a $\mathcal{P}$ Maybe-coalgebra

- a partial Markov chain is a D-coalgebra.

There are also easy variants for labelled systems.

Lemma 1. [8] Let $\mathcal{C}$ be a category and $\mathcal{B}$ a reflective replete (i.e. full and isomorphism-closed) subcategory of $\mathcal{C}$.

1. Let $A \in$ ob $\mathcal{C}$. Then $A$ is a final object of $\mathcal{C}$ iff it is a final object of $\mathcal{B}$.

2. Let $F$ be an endofunctor on $\mathcal{C}$. Then $\operatorname{Coalg}(\mathcal{B}, F)$ is a reflective replete subcategory of $\operatorname{Coalg}(\mathcal{C}, F)$.

Examples of reflective replete subcategories:

- Poset of Preord, and DiscSetoid of Setoid. In each case the reflection is given by $Q$ with unit $p$.

- Setoid of Preord. At $A$, the reflection is $\left(A_{0}, \equiv\right)$, where $\equiv$ is the least equivalence relation containing $\leqslant_{A}$, with unit $\mathrm{id}_{A_{0}}$.

\section{Relators}

\subsection{Relators and Simulation}

Any notion of simulation depends on a way of transforming a relation. For example, given a relation $X \stackrel{\mathcal{R}}{\longrightarrow} Y$, we define

$-\mathcal{P} X \stackrel{\operatorname{Sim} \mathcal{R}}{\longrightarrow} \mathcal{P} Y$ to relate $u$ to $v$ when $\forall x \in u . \exists y \in v . x \mathcal{R} y$

$-\mathcal{P} X \stackrel{\text { Bisim } \mathcal{R}}{\longrightarrow} \mathcal{P} Y$ to relate $u$ to $v$ when $\forall x \in u . \exists y \in v . x \mathcal{R} y$ and $\forall y \in v . \exists x \in u . x \mathcal{R} y$.

for simulation and bisimulation respectively. In general:

Definition 6. Let $F$ be an endofunctor on Set. An F-relator maps each relation $X \stackrel{\mathcal{R}}{\longrightarrow} Y$ to a relation $F X \stackrel{\Gamma \mathcal{R}}{\longrightarrow} F Y$ in such a way that the following hold.

- For any relations $X \stackrel{\mathcal{R}, \mathcal{S}}{\longrightarrow} Y$, if $\mathcal{R} \subseteq \mathcal{S}$ then $\Gamma \mathcal{R} \subseteq \Gamma \mathcal{S}$.

- For any set $X$ we have $\left(=_{F X}\right) \subseteq \Gamma\left(=_{X}\right)$

- For any relations $X \stackrel{\mathcal{R}}{\longrightarrow} Y \stackrel{\mathcal{S}}{\longrightarrow} Z$ we have $(\Gamma \mathcal{R}) ;(\Gamma \mathcal{S}) \subseteq \Gamma(\mathcal{R} ; \mathcal{S})$

- For any functions $Z \stackrel{f}{\longrightarrow} X$ and $W \stackrel{g}{\longrightarrow} Y$, and any relation $X \stackrel{\mathcal{R}}{\longrightarrow} Y$, we have $\Gamma(f, g)^{-1} \mathcal{R}=(F f, F g)^{-1} \Gamma \mathcal{R}$.

An $F$-relator $\Gamma$ is conversive when $\Gamma\left(\mathcal{R}^{\mathrm{c}}\right)=(\Gamma \mathcal{R})^{\mathrm{c}}$ for every relation $X \stackrel{\mathcal{R}}{\longrightarrow} Y$. 
For example: Sim is a $\mathcal{P}$-relator, and Bisim is a conversive $\mathcal{P}$-relator.

We can now give a general definition of simulation.

Definition 7. Let $F$ be an endofunctor on Set, and let $\Gamma$ be an F-relator. Let $M$ and $N$ be $F$-coalgebras.

1. A $\Gamma$-simulation from $M$ to $N$ is a relation $M \stackrel{\mathcal{R}}{\longrightarrow} N$ such that $\mathcal{R} \subseteq$ $\left(\zeta_{M}, \zeta_{N}\right)^{-1} \Gamma \mathcal{R}$

2. The largest $\Gamma$-simulation is called $\Gamma$-similarity and written $\lesssim_{M, N}$.

3. $M$ is $\Gamma$-encompassed by $N$, written $M \preccurlyeq^{\Gamma} N$, when for every $x \in M$ there is $y \in N$ such that $x \lesssim_{M, N} y$ and $y \lesssim_{N, M} x$.

For example: a Sim-simulation is an ordinary simulation, and a Bisim-simulation is a bisimulation.

The basic properties of simulations are as follows.

Lemma 2. Let $F$ be an endofunctor on Set, and $\Gamma$ an $F$-relator.

1. Let $M$ be an F-coalgebra. Then $M \stackrel{\left(=_{M}\right)}{\longrightarrow} M$ is a $\Gamma$-simulation. Moreover $\lesssim_{M, M}$ is a preorder on $M \cdot$-an equivalence relation if $\Gamma$ is conversive.

2. Let $M, N, P$ be F-coalgebras. If $M \stackrel{\mathcal{R}}{\longrightarrow} N \stackrel{\mathcal{S}}{\longrightarrow} P$ are $\Gamma$-simulations then so is $M \stackrel{\mathcal{R} ; \mathcal{S}}{\longrightarrow} P$. Moreover $\left(\lesssim_{M, N}^{\Gamma}\right) ;\left(\lesssim_{N, P}^{\Gamma}\right) \sqsubseteq\left(\lesssim_{M, P}^{\Gamma}\right)$.

3. Let $M$ and $N$ be $F$-coalgebras, and let $\Gamma$ be conversive. If $M \stackrel{\mathcal{R}}{\longrightarrow} N$ is a simulation then so is $N \stackrel{\mathcal{R}^{\mathrm{c}}}{\longrightarrow} M$. Moreover $\left(\lesssim_{M, N}^{\Gamma}\right)^{\mathrm{c}}=\left(\lesssim_{N, M}^{\Gamma}\right)$ and $\lesssim_{M, N}$ is difunctional.

4. Let $M \stackrel{f}{\longrightarrow} N$ and $M^{\prime} \stackrel{g}{\longrightarrow} N^{\prime}$ be F-coalgebra morphisms. If $N \stackrel{\mathcal{R}}{\longrightarrow} N^{\prime}$ is a $\Gamma$-simulation then so is $M \stackrel{(f, g)^{-1} \mathcal{R}}{\longrightarrow} M^{\prime}$. Moreover $(f, g)^{-1}\left(\lesssim_{N, N^{\prime}}\right)=$ $\left(\lesssim \Gamma, M^{\prime}\right)$.

5. $\preccurlyeq^{\Gamma}$ is a preorder on the class of $F$-coalgebras.

6. Let $M \stackrel{f}{\longrightarrow} N$ be an F-coalgebra morphism. Then $x$ and $f(x)$ are mutually $\Gamma$-similar for all $x \in M$. Hence $M \preccurlyeq N$, and if $f$ is surjective then also $N \preccurlyeq M$.

An $F$-coalgebra is all- $\Gamma$-encompassing when it is greatest in the $\preccurlyeq^{\Gamma}$ preorder. For example, take the disjoint union of all transition systems carried by an initial segment of $\mathbb{N}$. This is an all-Bisim-encompassing $\mathcal{P}^{\left[0, \aleph_{0}\right]}$-coalgebra, because every

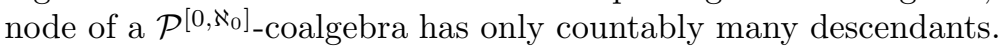

\subsection{Relators Preserving Binary Composition}

Definition 8. Let $F$ be an endofunctor on Set. An F-relator $\Gamma$ is said to preserve binary composition when for all sets $X, Y, Z$ and relations $X \stackrel{\mathcal{R}}{\longrightarrow} Y \stackrel{\mathcal{S}}{\longrightarrow} Z$ we have $\Gamma(\mathcal{R} ; \mathcal{S})=(\Gamma \mathcal{R}) ;(\Gamma \mathcal{S})$. If we also have $\Gamma\left(=_{X}\right)=$ $\left(=_{F X}\right)$ for every set $X$, then $F$ is functorial. 
For example, Sim preserves binary composition and Bisim is functorial. We shall examine relators preserving binary composition using the following notions.

\section{Definition 9}

1. A commutative square $Z \stackrel{g}{\longrightarrow} Y$ in Set is a quasi-pullback when

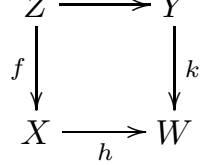

$$
\forall x \in X . \forall y \in Y . h(x)=k(y) \Rightarrow \exists z \in Z . x=f(z) \wedge g(z)=y
$$

2. A commutative square $C \stackrel{g}{\longrightarrow} B$ in Preord is a preorder-quasi-pullback

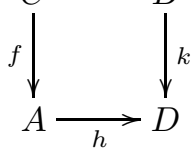

when $\forall x \in A . \forall y \in B . h(x) \leqslant_{D} k(y) \Rightarrow \exists z \in C . x \leqslant_{A} f(z) \wedge g(z) \leqslant_{B} y$

Definition 10. (adapted from [13]) Let $F$ be an endofunctor on Set. A stable preorder on $F$ is a functor $G:$ Set $\longrightarrow$ Preord that makes

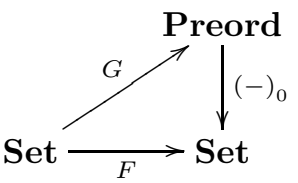

commute and sends quasi-pullbacks to preorder-quasi-pullbacks. It is a stable equivalence relation on $F$ when it is a functor Set $\longrightarrow$ Setoid.

For any relation $X \stackrel{\mathcal{R}}{\longrightarrow} Y$, we write $X \stackrel{\pi_{\mathcal{R}}}{\longleftarrow} \mathcal{R} \stackrel{\pi_{\mathcal{R}}^{\prime}}{\longrightarrow} Y$ for the two projections. We can now give our main result.

Theorem 1. Let $F$ be an endofunctor on Set. There is a bijection between

- F-relators preserving binary composition

- stable preorders on $F$

described as follows.

- Given an F-relator $\Gamma$ preserving binary composition, we define the stable preorder $\tilde{\Gamma}$ on $F$ to map $X$ to $\left(F X, \Gamma\left(=_{X}\right)\right)$ and $X \stackrel{f}{\longrightarrow} Y$ to $F f$.

- Given a stable preorder $G$ on $F$, we define the $F$-relator $\hat{G}$ to map a relation $X \stackrel{\mathcal{R}}{\longrightarrow} Y$ to

$$
\left\{(x, y) \in F X \times F Y \mid \exists z \in F \mathcal{R} . x \leqslant_{G X}\left(F \pi_{\mathcal{R}}\right) z \wedge\left(F \pi_{\mathcal{R}}^{\prime}\right) z \leqslant_{G Y} y\right\}
$$

It restricts to a bijection between

- conversive F-relators preserving binary composition

- stable equivalence relations on $F$. 
Corollary 1. [3] Let $F$ be an endofunctor on Set.

1. Suppose $F$ preserves quasi-pullbacks. Then we obtain a conversive functorial F-relator $\hat{F}$ mapping a relation $X \stackrel{\mathcal{R}}{\longrightarrow} Y$ to

$$
\left\{(x, y) \in F X \times F Y \mid \exists z \in F \mathcal{R} . x=\left(F \pi_{\mathcal{R}}\right) z \wedge\left(F \pi_{\mathcal{R}}^{\prime}\right) z=y\right\}
$$

2. Let $\Gamma$ be a functorial $F$-relator. Then $F$ preserves quasi-pullbacks and $\Gamma=\hat{F}$.

\subsection{Further Examples of Relators}

We first note several ways of constructing relators.

Lemma 3. 1. Let $F$ be an endofunctor on Set, and $\left(\Gamma_{j}\right)_{j \in J}$ a family of $F$ relators. Then

$$
\prod_{j \in J} \Gamma_{j}:(X \stackrel{\mathcal{R}}{\longrightarrow} Y) \mapsto \bigcap_{j \in J} \Gamma_{j} \mathcal{R}
$$

is an F-relator. If $M$ and $N$ are F-coalgebras, then $M \stackrel{\mathcal{R}}{\longrightarrow} N$ is a $\prod_{j \in J} \Gamma_{j}$-simulation from $M$ to $N$ iff, for all $j \in J$, it is a $\Gamma_{j}$-simulation from $M$ to $N$.

2. Let $F$ be an endofunctor on Set, and $\Gamma$ an $F$-relator. Then

$$
\Gamma^{\mathrm{c}}:(X \stackrel{\mathcal{R}}{\longrightarrow} Y) \mapsto\left(\Gamma \mathcal{R}^{\mathrm{c}}\right)^{\mathrm{c}}
$$

is an F-relator. If $M$ and $N$ are F-coalgebras, then $M \stackrel{\mathcal{R}}{\longrightarrow} N$ is a $\Gamma^{\mathrm{C}_{-}}$ simulation from $M$ to $N$ iff $\mathcal{R}^{\mathrm{c}}$ is a $\Gamma$-simulation from $N$ to $M$; hence $\left(\lesssim_{M, N}^{\mathrm{c}}\right)=\left(\lesssim_{N, M}\right)^{\mathrm{c}}$

3. Let $F$ and $G$ be endofunctors on Set and $F \stackrel{\alpha}{\longrightarrow} G$ a natural transformation. Let $\Gamma$ be an $G$-relator. Then

$$
\alpha^{-1} \Gamma:(X \stackrel{\mathcal{R}}{\longrightarrow} Y) \mapsto\left(\alpha_{X}, \alpha_{Y}\right)^{-1} \Gamma \mathcal{R}
$$

is an F-relator. If $M$ and $N$ are F-coalgebras, then $M \stackrel{\mathcal{R}}{\longrightarrow} N$ is an $\alpha^{-1} \Gamma$-simulation from $M$ to $N$ iff it is a $\Gamma$-simulation from $\operatorname{Coalg}(\operatorname{Set}, \alpha) M$ to $\operatorname{Coalg}(\operatorname{Set}, \alpha) N$; hence $\left(\lesssim_{M, N}^{\alpha^{-1} \Gamma}\right)=\left(\lesssim_{\operatorname{Coalg}(\operatorname{Set}, \alpha) M, \operatorname{Coalg}(\operatorname{Set}, \alpha) N}\right)$.

4. The identity operation on relations is an $\mathrm{id}_{\mathbf{S e t}}$-relator.

5. Let $F$ and $F^{\prime}$ be endofunctors on Set. If $\Gamma$ is an $F$-relator and $\Gamma^{\prime}$ an $F^{\prime}$ relator, then $\Gamma^{\prime} \Gamma$ is an $F^{\prime} F$-relator.

Note that $\Gamma \sqcap \Gamma^{\mathrm{c}}$ is the greatest conversive relator contained in $\Gamma$.

We give some relators for our examples:

- Via Def. 3(3) , Sim and Bisim are $\mathcal{P}^{[0, \kappa)}$-relators and $\mathcal{P}^{[1, \kappa)}$-relators where $\kappa$ is a cardinal or $\infty$. Moreover Sim preserves binary composition, and if $\kappa \leqslant 3$ or $\kappa \geqslant \aleph_{0}$ then Bisim is functorial. But for $4 \leqslant \kappa<\aleph_{0}$, the functors $\mathcal{P}^{[0, \kappa)}$ and $\mathcal{P}^{[1, \kappa)}$ do not preserve quasi-pullbacks, so Bisim does not preserve binary composition over them. 
- We define $\mathcal{P}$ Maybe-relators, all preserving binary composition. For a relation $X \stackrel{\mathcal{R}}{\longrightarrow} Y$,

$$
\begin{aligned}
& \text { LowerSim } \mathcal{R} \stackrel{\text { def }}{=}\{(u, v) \in \mathcal{P} \text { Maybe } X \times \mathcal{P} \text { Maybe } Y \mid \\
& \left.\forall x \in \mathrm{Just}^{-1} u . \exists y \in \mathrm{Just}^{-1} v .(x, y) \in \mathcal{R}\right\} \\
& \operatorname{UpperSim} \mathcal{R} \stackrel{\text { def }}{=}\{(u, v) \in \mathcal{P} \text { Maybe } X \times \mathcal{P} \text { Maybe } Y \mid \Uparrow \notin u \Rightarrow \\
& \Uparrow \notin v \\
& \left.\left.\wedge \forall y \in \mathrm{Just}^{-1} v . \exists x \in \mathrm{Just}^{-1} u .(x, y) \in \mathcal{R}\right)\right\} \\
& \text { ConvexSim } \stackrel{\text { def }}{=} \text { LowerSim } \sqcap \text { UpperSim } \\
& \operatorname{SmashSim} \mathcal{R} \stackrel{\text { def }}{=}\{(u, v) \in \mathcal{P} \text { Maybe } X \times \mathcal{P} \text { Maybe } Y \mid \Uparrow \notin u \Rightarrow \\
& \Uparrow \notin v \\
& \wedge \forall y \in \text { Just }^{-1} v . \exists x \in \text { Just }^{-1} u .(x, y) \in \mathcal{R} \\
& \left.\wedge \forall x \in \text { Just }^{-1} u . \exists y \in \text { Just }^{-1} v .(x, y) \in \mathcal{R}\right\} \\
& \text { InclusionSim } \mathcal{R} \stackrel{\text { def }}{=}\{(u, v) \in \mathcal{P} \text { Maybe } X \times \mathcal{P} \text { Maybe } Y \mid \\
& \left.\forall x \in \mathrm{Just}^{-1} u . \exists y \in \mathrm{Just}^{-1} v .(x, y) \in \mathcal{R}\right\} \\
& \wedge \Uparrow \in u \Rightarrow \Uparrow \in v\}
\end{aligned}
$$

We respectively obtain notions of lower, upper, convex, smash and inclusion simulation on transiton systems with divergence 10 20]. By taking converses and intersections of these relators, we obtain-besides $T$-nineteen different relators of which three are conversive. A more systematic analysis that includes these is presented in [16].

- We define $D$-relators. For a relation $X \stackrel{\mathcal{R}}{\longrightarrow} Y$

$$
\begin{aligned}
\operatorname{ProbSim} \mathcal{R} & \stackrel{\text { def }}{=}\left\{\left(d, d^{\prime}\right) \in D X \times D Y \mid \forall U \subseteq X . d U \leqslant d^{\prime} \mathcal{R}(U)\right\} \\
\operatorname{ProbBisim} \mathcal{R} & \stackrel{\text { def }}{=}\left\{\left(d, d^{\prime}\right) \in D X \times D Y \mid \forall U \subseteq X . d U \leqslant d^{\prime} \mathcal{R}(U) \wedge d(\Uparrow) \leqslant d^{\prime}(\Uparrow)\right\}
\end{aligned}
$$

where $\mathcal{R}(U) \stackrel{\text { def }}{=}\{y \in Y \mid \exists x \in U .(x, y) \in \mathcal{R}\}$. In fact ProbBisim is the greatest conversive relator contained in ProbSim. We obtain notions of simulation and bisimulation on partial Markov chains as in 56/21]5/22. By Thm. 1 of 14], ProbSim preserves binary composition and ProbBisim is functorial.

\section{Theory of Simulation and Final Coalgebras}

Throughout this section, $F$ is an endofunctor on Set and $\Gamma$ is an $F$-relator.

\section{1 $Q F_{\Gamma}$-Coalgebras}

Definition 11. $F_{\Gamma}$ is the endofunctor on Preord that maps $A$ to $\left(F A_{0}, \Gamma\left(\leqslant_{A}\right)\right)$ and $A \stackrel{f}{\longrightarrow} B$ to $F f$. 
Thus we obtain an endofunctor $Q F_{\Gamma}$ on Preord. It restricts to Poset and also, if $\Gamma$ is conversive, to Setoid and to DiscSetoid.

For example, if $A$ is a preordered set, then $Q \mathcal{P}_{\operatorname{Sim}}^{\left[0, \aleph_{0}\right]} A$ is (isomorphic to) the set of countably generated lower sets, ordered by inclusion. The probabilistic case is unusual: $D_{\text {Probsim }}$ is already an endofunctor on Poset, so applying $Q$ makes no difference (up to isomorphism). This reflects the fact that, for partial Markov chains, mutual similarity is bisimilarity [6].

A $Q F_{\Gamma^{-}}$coalgebra $M$ is said to be final when the following equivalent conditions hold:

- $M$ is final in Coalg(Preord, $\left.Q F_{\Gamma}\right)$

- $M$ is final in Coalg(Poset, $\left.Q F_{\Gamma}\right)$.

If $\Gamma$ is conversive, the following are equivalent to the above:

- $M$ is final in Coalg(Setoid, $\left.Q F_{\Gamma}\right)$

$-M$ is final in Coalg(DiscSetoid, $\left.Q F_{\Gamma}\right)$.

These equivalences follow from Lemma 1.

We adapt Def. 7 and Lemma 2 from $F$-coalgebras to $Q F_{\Gamma^{-}}$-coalgebras.

Definition 12. Let $M$ and $N$ be $Q F_{\Gamma}$-coalgebras.

1. A simulation from $M$ to $N$ is a bimodule $M \stackrel{\mathcal{R}}{\longrightarrow} N$ such that $\mathcal{R} \subseteq$ $\left(\zeta_{M}, \zeta_{N}\right)^{-1} Q \Gamma \mathcal{R}$.

2. The greatest simulation is called similarity and written $\lesssim_{M, N}$.

3. $M$ is encompassed by $N$, written $M \preccurlyeq N$, when for every $x \in M$ there is $y \in N$ such that $x \lesssim_{M, N} y$ and $y \lesssim_{N, M} x$.

Lemma 4. Let $F$ be an endofunctor on Set, and $\Gamma$ an $F$-relator.

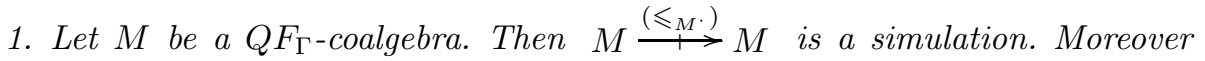
$\lesssim_{M, M}$ is a preorder on $M_{0}^{\cdot}$-an equivalence relation if $\Gamma$ is conversive - that contains $\leqslant_{M}$.

2. Let $M, N, P$ be $Q F_{\Gamma}$-coalgebras. If $M \stackrel{\mathcal{R}}{\longrightarrow} N \stackrel{\mathcal{S}}{\longrightarrow} P$ are simulations then so is $M \stackrel{\mathcal{R} ; \mathcal{S}}{\longrightarrow} P$. Moreover $\left(\lesssim_{M, N}\right) ;\left(\lesssim_{N, P}\right) \sqsubseteq\left(\lesssim_{M, P}\right)$.

3. Let $M$ and $N$ be $Q F_{\Gamma^{-}}$-coalgebras, and let $\Gamma$ be conversive. If $M \stackrel{\mathcal{R}}{\longrightarrow} N$ is a simulation then so is $N \stackrel{\overline{\mathcal{R}^{c}}}{\longrightarrow} M$ - recall that this is $\left(\leqslant_{N^{*}}\right) ; \mathcal{R}^{\mathrm{c}} ;\left(\leqslant_{M^{*}}\right)$. Moreover $\left(\lesssim_{M, N}\right)^{\mathrm{c}}=\left(\lesssim_{N, M}\right)$ and $\lesssim_{M, N}$ is difunctional.

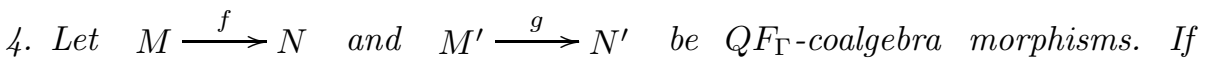
$N \stackrel{\mathcal{R}}{\longrightarrow} N^{\prime}$ is a simulation then so is $M \stackrel{(f, g)^{-1} \mathcal{R}}{\longrightarrow} M^{\prime}$. Moreover $\left(\lesssim_{M, M^{\prime}}\right)=(f, g)^{-1}\left(\lesssim_{N, N^{\prime}}\right)$.

5. $\preccurlyeq$ is a preorder on the class of $Q F_{\Gamma}$-coalgebras. 
6. Let $M \stackrel{f}{\longrightarrow} N$ be an $Q F_{\Gamma}$-coalgebra morphism. Then $x$ and $f(x)$ are $m u$ tually similar for all $x \in M^{*}$. Hence $M \preccurlyeq N$, and if $f$ is surjective then also $N \preccurlyeq M$.

We can also characterize coalgebra morphisms.

Lemma 5. Let $M$ and $N$ be $Q F_{\Gamma}$-coalgebras. For any function $M_{0}^{\cdot} \stackrel{f}{\longrightarrow} N_{0}$, the following are equivalent.

1. $M \stackrel{f}{\longrightarrow} N$ is a $Q F_{\Gamma}$-coalgebra morphism.

2. $M \stackrel{\left(f, N_{0}^{\cdot}\right)^{-1}\left(\leqslant_{N^{*}}\right)}{\longrightarrow} N$ and $N \stackrel{\left(N_{0}^{\cdot}, f\right)^{-1}\left(\leqslant_{N^{*}}\right)}{\longrightarrow} M$ are both simulations.

A $Q F_{\Gamma}$-coalgebra $N$ is all-encompassing when it is encompasses every $M \in \operatorname{Coalg}\left(\right.$ Preord, $\left.Q F_{\Gamma}\right)$, or equivalently every $M \in \operatorname{Coalg}\left(\right.$ Poset, $\left.Q F_{\Gamma}\right)$, or equivalently - if $\Gamma$ is conversive every $M \in \operatorname{Coalg}\left(\right.$ Setoid, $\left.Q F_{\Gamma}\right)$ or every $M \in$ Coalg(Setoid, $Q F_{\Gamma}$ ). These equivalences follow from the surjectivity of the units of the reflections.

\subsection{Extensional Coalgebras}

Definition 13. An extensional coalgebra is $M \in \operatorname{Coalg}\left(\right.$ Poset, $\left.Q F_{\Gamma}\right)$ such that $\left(\lesssim_{M, M}\right)=\left(\leqslant_{M}\right)$. We write ExtCoalg $(\Gamma)$ for the category of extensional coalgebras and coalgebra morphisms.

These coalgebras enjoy several properties.

Lemma 6. Let $N$ be an extensional coalgebra.

1. If $\Gamma$ is conversive, then $N \cdot$ is a discrete setoid.

2. Let $M$ be a $Q F_{\Gamma}$-coalgebra and $N \stackrel{f}{\longrightarrow} M$ a coalgebra morphism. Then $f$ is order-reflecting and injective.

3. Let $M$ be a $Q F_{\Gamma}$-coalgebra and $M \stackrel{f}{\longrightarrow} N$ an order-reflecting, injective coalgebra morphism. Then $M$ is extensional.

4. Let $M$ be a $Q F_{\Gamma}$-coalgebra such that $M \preccurlyeq N$. Then there is a unique $Q F_{\Gamma^{-}}$ coalgebra morphism $M \stackrel{f}{\longrightarrow} N$.

Thus ExtCoalg $(\Gamma)$ is just a preordered class. It is a replete subcategory of Coalg $\left(\right.$ Poset, $\left.Q F_{\Gamma}\right)$ and also - if $\Gamma$ is conversive - of Coalg(DiscSetoid, $\left.Q F_{\Gamma}\right)$. We next see that is reflective within $\operatorname{Coalg}\left(\operatorname{Preord}, Q F_{\Gamma}\right)$.

Lemma 7. (Extensional Quotient) Let $M$ be a $Q F_{\Gamma}$-coalgebra, and define $\mathbf{p}_{M} \stackrel{\text { def }}{=}$ $p_{\left(M_{0}, \lesssim_{M, M}\right)}$.

1. There is a $Q F_{\Gamma}$-coalgebra $\mathbf{Q} M$ carried by $Q\left(M_{0}^{\cdot}, \lesssim_{M, M}\right)$, uniquely characterized by the fact that $M \stackrel{\mathbf{p}_{M}}{\longrightarrow} \mathbf{Q} M$ is a coalgebra morphism. 
2. $\mathbf{Q} M$, with unit $\mathbf{p}_{M}$, is a reflection of $M$ in $\operatorname{ExtCoalg}(\Gamma)$.

More generally, a $Q F_{\Gamma}$-coalgebra $M$ can be quotiented by any $\left(\leqslant_{M} \cdot\right)$-containing preorder that is an endosimulation on $M$; but we shall not need this.

Lemma 8. Let $M$ be a $Q F_{\Gamma}$-coalgebra. The following are equivalent.

1. $M$ is a final $Q F_{\Gamma}$-coalgebra.

2. $M$ is all-encompassing and extensional.

3. $M$ is extensional, and encompasses all extensional $Q F_{\Gamma}$-coalgebras.

Lemma 9. Let $M$ be a $Q F_{\Gamma}$-coalgebra. The following are equivalent.

1. $M$ is all-encompassing.

2. $M$ encompasses all extensional coalgebras.

3. $\mathbf{Q} M$ is a final $Q F_{\Gamma}$-coalgebra.

\subsection{Relating $F$-Coalgebras and $Q F_{\Gamma}$-Coalgebras}

We have studied $F$-coalgebras and $Q F_{\Gamma}$-coalgebras separately, but now we connect them: each $F$-coalgebra gives rise to a $Q F_{\Gamma^{-}}$-coalgebra, and the converse is also true in a certain sense.

Definition 14. The functor $\Delta^{\Gamma}: \operatorname{Coalg}($ Set,$F) \longrightarrow \operatorname{Coalg}\left(\right.$ Preord, $\left.Q F_{\Gamma}\right)$ maps

- an F-coalgebra $M=\left(M^{*}, \zeta_{M}\right)$ to the $Q F_{\Gamma}$-coalgebra with carrier $\Delta M^{\cdot}$ and structure $\Delta M \stackrel{\zeta_{M}}{\longrightarrow} F_{\Gamma} \Delta M \stackrel{p_{F_{\Gamma} \Delta M^{*}}^{\longrightarrow}}{\longrightarrow} Q F_{\Gamma} \Delta M$.

- an F-coalgebra morphism $M \stackrel{f}{\longrightarrow} N$ to $f$.

Lemma 10. Let $M$ and $N$ be $F$-coalgebras. Then a $\Gamma$-simulation from $M$ to $N$ is precisely a simulation from $\Delta^{\Gamma} M$ to $\Delta^{\Gamma} N$. Hence $\left(\lesssim_{\Delta^{\Gamma} M, \Delta \Gamma N}\right)=\left(\lesssim_{M, N}\right)$, and $M \preccurlyeq \preccurlyeq^{\Gamma} N$ iff $\Delta^{\Gamma} M \preccurlyeq \Delta^{\Gamma} N$.

We are thus able to use a final $Q F_{\Gamma}$-coalgebra to characterize similarity in $F$ coalgebras.

Theorem 2. Let $M$ be a final $Q F_{\Gamma}$-coalgebra; for any $Q F_{\Gamma^{-}}$-coalgebra $P$ we write $P \stackrel{a_{P}}{\longrightarrow} M$ for its anamorphism. Let $N$ and $N^{\prime}$ be $F$-coalgebras. Then

$$
\left(\lesssim_{N, N^{\prime}}\right)=\left(a_{\Delta \Gamma N}, a_{\Delta \Gamma N^{\prime}}\right)^{-1}\left(\leqslant_{M^{*}}\right)
$$

Our other results require moving from a $Q F_{\Gamma^{-}}$coalgebra to an $F$-coalgebra.

Lemma 11. Let $M$ be a $Q F_{\Gamma}$-coalgebra. Then there is an $F$-coalgebra $N$ and a surjective $Q F_{\Gamma}$-coalgebra morphism $\Delta^{\Gamma} N \stackrel{f}{\longrightarrow} M$.

\section{Theorem 3}

1. Let $M$ be an $F$-coalgebra. Then $\mathbf{Q} \Delta^{\Gamma} M$ is a final $Q F_{\Gamma^{-}}$-coalgebra iff $M$ is all- $\Gamma$-encompassing.

2. Any final $Q F_{\Gamma^{-}}$-coalgebra is isomorphic to one of this form. 


\section{Beyond Similarity}

\section{$5.1 \quad$ Multiple Relations}

We recall from [9] that a 2-nested simulation from $M$ to $N$ (transition systems) is a simulation contained in the converse of similarity. Let us say that a nested preordered set is a set equipped with two preorders $\leqslant_{n}$ (think 2-nested similarity) and $\leqslant_{0}$ (think converse of similarity) such that $\left(\leqslant_{n}\right) \subseteq\left(\leqslant_{0}\right)$ and $\left(\leqslant_{n}\right) \subseteq\left(\geqslant_{0}\right)$. It is a nested poset when $\leqslant_{\mathrm{n}}$ is a partial order. By working with these instead of preordered sets and posets, we can obtain a characterization of 2-nested similarity as a final coalgebra.

We fix a set $I$. For our example of 2-nested simulation, it would be $\{\mathrm{n}, \mathrm{o}\}$.

Definition 15. (I-relations)

1. For any sets $X$ and $Y$, an $I$-relation $X \stackrel{\mathcal{R}}{\longrightarrow} Y$ is an $I$-indexed family $\left(\mathcal{R}_{i}\right)_{i \in I}$ of relations from $X$ to $Y$. We write $\operatorname{Rel}_{I}(X, Y)$ for the complete lattice of I-relations ordered pointwise.

2. Identity I-relations $\left(=_{X}\right)$ and composite $I$-relations $\mathcal{R} ; \mathcal{S}$ are defined pointwise, as are inverse image $I$-relations $(f, g)^{-1} \mathcal{R}$ for functions $f$ and $g$.

We then obtain analogues of Def. 2 and 3. In particular, an I-preordered set $A$ is a set $A_{0}$ equipped with an $I$-indexed family of preorders $\left(\leqslant_{A, i}\right)_{i \in I}$, and it is an $I$-poset when $\bigcap_{i \in I}\left(\leqslant_{i}\right)$ is a partial order. We thus obtain categories Preord Pr $_{I}$ and Poset $_{I}$, whose morphisms are monotone functions, i.e. monotone in each component. Given an $I$-preordered set $A$, the principal lower set of $x \in A$ is $\left\{y \in A \mid \forall i \in I . y \leqslant_{A, i} x\right\}$. The quotient I-poset $Q A$ is $\left\{[x]_{A} \mid x \in A\right\}$ with $i$ th preorder relating $[x]_{A}$ to $[y]_{A}$ iff $x \leqslant A, i$, and we write $A \stackrel{p_{A}}{\longrightarrow} Q A$ for the function $x \mapsto[x]_{A}$. Thus Poset $_{I}$ is a reflective replete subcategory of $\operatorname{Preord}_{I}$.

Returning to our example, a nested preordered set is a $\{\mathrm{n}, \mathrm{o}\}$-preordered set, subject to some constraints that we ignore until Sect. 5.2.

For the rest of this section, let $F$ be an endofunctor on Set, and $\Lambda$ an $F$ relator $I$-matrix, i.e. an $I \times I$-indexed family of $F$-relators $\left(\Lambda_{i, j}\right)_{i, j \in I}$. This gives us an operation on $I$-relations as follows.

Definition 16. For any I-relation $F X \stackrel{\mathcal{R}}{\longrightarrow} F Y$, we define the I-relation $F X \stackrel{\Lambda \mathcal{R}}{\longrightarrow} F Y$ as $\left(\bigcap_{j \in I} \Lambda_{i, j} \mathcal{R}_{j}\right)_{i \in I}$.

For our example, we take the $\mathcal{P}$-relator $\{\mathrm{n}, \mathrm{o}\}$-matrix TwoSim

$$
\begin{array}{ll}
\operatorname{TwoSim}_{n, n} \stackrel{\text { def }}{=} \operatorname{Sim} & \text { TwoSim }_{\mathrm{n}, \mathrm{o}} \stackrel{\text { def }}{=} \operatorname{Sim}^{c} \\
\text { TwoSim }_{\mathrm{o}, \mathrm{n}} \stackrel{\text { def }}{=} T & \text { TwoSim }_{\mathrm{o}, \mathrm{o}} \stackrel{\text { def }}{=} \operatorname{Sim}^{c}
\end{array}
$$

We can see that the operation $\mathcal{R} \mapsto \Lambda \mathcal{R}$ has the same properties as a relator.

\section{Lemma 12}

1. For any I-relations $X \stackrel{\mathcal{R}, \mathcal{S}}{\longrightarrow} Y$, if $\mathcal{R} \sqsubseteq \mathcal{S}$ then $\Lambda \mathcal{R} \sqsubseteq \Lambda \mathcal{S}$.

2. For any set $X$ we have $\left(=_{F X}\right) \sqsubseteq \Lambda\left(=_{X}\right)$ 
3. For any I-relations $X \stackrel{\mathcal{R}}{\longrightarrow} Y \stackrel{\mathcal{S}}{\longrightarrow} Z$ we have $(\Lambda \mathcal{R}) ;(\Lambda \mathcal{S}) \sqsubseteq \Lambda(\mathcal{R} ; \mathcal{S})$

4. For any functions $X^{\prime} \stackrel{f}{\longrightarrow} X$ and $Y^{\prime} \stackrel{g}{\longrightarrow} Y$ and any I-relation $X \stackrel{\mathcal{R}}{\longrightarrow} Y$, we have $\Lambda(f, g)^{-1} \mathcal{R}=(F f, F g)^{-1} \Lambda \mathcal{R}$.

Note by the way that TwoSim as a $\mathcal{P}$-relator matrix does not preserve binary composition. Now we adapt Def. 7.

Definition 17. Let $M$ and $N$ be F-coalgebras.

1. A $\Lambda$-simulation from $M$ to $N$ is an $I$-relation $M \stackrel{\mathcal{R}}{\longrightarrow} N$ such that for all $i, j \in I$ we have $\mathcal{R}_{i} \in\left(\zeta_{M}, \zeta_{N}\right)^{-1} \Lambda_{i, j} \mathcal{R}_{j}$, or equivalently $\mathcal{R} \sqsubseteq \Lambda\left(\zeta_{M}, \zeta_{N}\right)^{-1} \mathcal{R}$.

2. The largest $\Lambda$-simulation is called $\Lambda$-similarity and written $\lesssim \Lambda, N$.

3. $N$ is said to $\Lambda$-encompass $M$ when for every $x \in M$ there is $y \in N$ such that, for all $i \in I$, we have $x\left(\lesssim_{M, N, i}\right)$ y and $y\left(\lesssim_{N, M, i}\right) x$.

In our example, the n-component of $\lesssim_{M, N}^{\mathrm{TwoSim}}$ is 2-nested similarity, and the o-component is the converse of similarity from $N$ to $M$.

The rest of the theory in Sect. 4 goes through unchanged, using Lemma 12.

\subsection{Constraints}

We wish to consider not all $I$-preordered sets (for a suitable indexing set $I$ ) but only those that satisfy certain constraints. These constraints are of two kinds:

- a "positive constraint" is a pair $(i, j)$ such that we require $\left(\leqslant_{i}\right) \subseteq\left(\leqslant_{j}\right)$

- a "negative constraint" is a pair $(i, j)$ such that we require $\left(\leqslant_{i}\right) \subseteq\left(\geqslant_{j}\right)$.

Furthermore the set of constraints should be "deductively closed". For example, if $\left(\leqslant_{i}\right) \subseteq\left(\geqslant_{j}\right)$ and $\left(\leqslant_{j}\right) \subseteq\left(\geqslant_{k}\right)$ then $\left(\leqslant_{i}\right) \subseteq\left(\leqslant_{k}\right)$.

Definition 18. A constraint theory on $I$ is a pair $\gamma=\left(\gamma^{+}, \gamma^{-}\right)$of relations on I such that $\gamma^{+}$is a preorder and $\gamma^{+} ; \gamma^{-} ; \gamma^{+} \subseteq \gamma^{-}$and $\gamma^{-} ; \gamma^{-} \subseteq \gamma^{+}$.

For our example, let $\gamma_{\text {nest }}$ be the constraint theory on $\{n, o\}$ given by

$$
\gamma_{\text {nest }}^{+}=\{(\mathrm{n}, \mathrm{n}),(\mathrm{n}, \mathrm{o}),(\mathrm{o}, \mathrm{o})\} \quad \gamma_{\text {nest }}^{-}=\{(\mathrm{n}, \mathrm{o})\}
$$

A constraint theory $\gamma$ gives rise to two operations $\gamma^{+L}$ and $\gamma^{-L}$ on relations (where $L$ stands for "lower adjoint"). They are best understood by seeing how they are used in the rest of Def. 19.

Definition 19. Let $\gamma$ be a constraint theory on I.

1. For an $I$-relation $X \stackrel{\mathcal{R}}{\longrightarrow} Y$, we define I-relations

$$
\begin{aligned}
& -X \underset{{ }_{1}^{+L} \mathcal{R}}{\longrightarrow} Y \text { as }\left(\bigcup_{j \in I(j, i) \in \gamma^{+}} \mathcal{R}_{j}\right)_{i \in I} \\
& -Y \stackrel{\gamma^{-L} \mathcal{R}}{\longrightarrow} X \text { as }\left(\bigcup_{j \in I(j, i) \in \gamma^{-}} \mathcal{R}_{j}^{\mathrm{c}}\right)_{i \in I}
\end{aligned}
$$


2. An I-endorelation $X \stackrel{\mathcal{R}}{\longrightarrow} X$ is $\gamma$-symmetric when

- for all $(j, i) \in \gamma^{+}$we have $\mathcal{R}_{j} \subseteq \mathcal{R}_{i}$, or equivalently $\gamma^{+L} \mathcal{R} \sqsubseteq \mathcal{R}$

- for all $(j, i) \in \gamma^{-}$we have $\mathcal{R}_{j}^{c} \subseteq \mathcal{R}_{i}$, or equivalently $\gamma^{-L} \mathcal{R} \sqsubseteq \mathcal{R}$.

3. We write Preord $_{\gamma}\left(\right.$ Poset $\left._{\gamma}\right)$ for the category of $\gamma$-symmetric I-preordered sets (I-posets) and monotone functions.

4. An I-relation $X \stackrel{\mathcal{R}}{\longrightarrow} Y$ is $\gamma$-difunctional when

- for all $(j, i) \in \gamma^{+}$we have $\mathcal{R}_{j} \subseteq \mathcal{R}_{i}$, or equivalently $\gamma^{+L} \mathcal{R} \sqsubseteq \mathcal{R}$

- for all $(j, i) \in \gamma^{-}$we have $\mathcal{R}_{i} ; \mathcal{R}_{j}^{\mathrm{c}} ; \mathcal{R}_{i} \subseteq \mathcal{R}_{i}$, or equivalently $\mathcal{R} ; \gamma^{-L} \mathcal{R} ; \mathcal{R} \sqsubseteq \mathcal{R}$

For our example, Preord $_{\gamma_{\text {nest }}}$ and Poset $_{\gamma_{\text {nest }}}$ are the categories of nested preordered sets and nested posets respectively. In general, Poset $\mathbf{P}_{\gamma}$ is a reflective replete subcategory of Preord $_{\gamma}$ and Preord $_{\gamma}$ of Preord $_{I}$.

Now let $F$ be an endofunctor and $\Lambda$ an $F$-relator $I$-matrix.

Definition 20. Let $\gamma$ be a constraint theory on I. Then $\Lambda$ is $\gamma$-conversive when

$$
\begin{aligned}
& \prod_{\substack{l \in I \\
l, k) \in \gamma^{+}}} \Lambda_{j, l} \sqsubseteq \Lambda_{i, k} \text { for all }(j, i) \in \gamma^{+} \text {and } k \in I \\
& \prod_{\substack{l \in I \\
(l, k) \in \gamma^{-}}} \Lambda_{j, l}^{c} \sqsubseteq \Lambda_{i, k} \text { for all }(j, i) \in \gamma^{-} \text {and } k \in I
\end{aligned}
$$

For our example, it is clear that the matrix TwoSim is $\gamma_{\text {nest-conversive. }}$

Lemma 13. Let $\gamma$ be a constraint theory on $I$ such that $\Lambda$ is $\gamma$-conversive. For every $I$-relation $X \stackrel{\mathcal{R}}{\longrightarrow} Y$ we have $\gamma^{+L} \Lambda \mathcal{R} \sqsubseteq \Lambda \gamma^{+L} \mathcal{R}$ and $\gamma^{-L} \Lambda \mathcal{R} \sqsubseteq \Lambda \gamma^{-L} \mathcal{R}$.

\subsection{Generalized Theory of Simulation and Final Coalgebras (Sketch)}

All the results of Sect. 4, in particular Thms. 2, 3, generalize to the setting of a set $I$ with a constraint theory $\gamma$. We replace "conversive" by " $\gamma$-conversive".

In our nested simulation example, we thus obtain an endofunctor $\mathcal{P}_{\text {TwoSim }}^{\left[0, \aleph_{0}\right]}$ on Preord Prest $_{\gamma_{\text {n }}}$ that maps a nested preordered set $A=\left(A_{0},\left(\leqslant_{A, \mathrm{n}}\right),\left(\leqslant_{A, \mathrm{o}}\right)\right)$ to $\left(\mathcal{P}^{\left[0, \aleph_{0}\right]} A_{0}, \operatorname{Sim}\left(\leqslant_{A, \mathrm{n}}\right) \cap \operatorname{Sim}^{\mathrm{c}}\left(\leqslant_{A, \mathrm{o}}\right), \operatorname{Sim}^{\mathrm{c}}\left(\leqslant_{A, \mathrm{o}}\right)\right)$. We conclude:

- (from Thm. 21) Given a final $Q \mathcal{P}_{\text {TwoSim }}^{\left[0, \aleph_{0}\right]}$-coalgebra $M$, we can use $\left(\leqslant_{M^{*}, \mathrm{n}}\right)$ and $\left(\geqslant_{M \cdot, o}\right)$ to characterize 2-nested similarity and similarity, respectively, in countably branching transition systems.

- (from Thm. 3) Given a countably branching transition system that is allBisim-encompassing (and hence all-TwoSim-encompassing), we can quotient it by 2 -nested similarity to obtain a final $Q \mathcal{P}_{\text {TwoSim }}^{\left[0, \aleph_{0}\right]}$-coalgebra.

Acknowledgements. I am grateful to Jean Goubault-Larrecq, Bartek Klin, Alexander Kurz, Sam Staton and James Worrell for their help. 


\section{References}

1. Aczel, P., Mendler, P.F.: A final coalgebra theorem. In: Dybjer, P., Pitts, A.M., Pitt, D.H., Poigné, A., Rydeheard, D.E. (eds.) Category Theory and Computer Science. LNCS, vol. 389, pp. 357-365. Springer, Heidelberg (1989)

2. Baltag, A.: A logic for coalgebraic simulation. ENTCS 33 (2000)

3. Carboni, A., Kelly, G.M., Wood, R.J.: A 2-categorical approach to change of base and geometric morphisms I. Cah. Topologie Géom. Différ. Catégoriques 32(1), 4795 (1991), http://www.numdam.org/item?id=CTGDC_1991__32_1_47_0

4. Cîrstea, C.: A modular approach to defining and characterising notions of simulation. Inf. Comput. 204(4), 469-502 (2006)

5. Danos, D., Laviolette, P.: Bisimulation and cocongruence for probabilistic systems. Information and Computation 204 (2006)

6. Desharnais, J.: A logical characterization of bisimulation for labelled Markov processes. In: Proceedings of the 2nd International Workshop on Probabilistic Methods in Verification, Univ. of Birmingham Technical Report CS-99-8, pp. 33-48 (1999)

7. Fábregas, I., de Frutos Escrig, D., Palomino, M.: Non-strongly stable orders also define interesting simulation relations. In: Kurz, A., Lenisa, M., Tarlecki, A. (eds.) CALCO 2009. LNCS, vol. 5728, pp. 221-235. Springer, Heidelberg (2009)

8. Freyd, P.J.: Algebraically complete categories. In: Proc. 1990 Como Category Theory Conf., Berlin. Lecture Notes in Mathematics, vol. 1488, pp. 95-104 (1991)

9. Groote, J.F., Vaandrager, F.: Structured operational semantics and bisimulation as a congruence. Information and Computation 100(2), 202-260 (1992)

10. Hennessy, M., Plotkin, G.D.: A term model for CCS. In: Dembiński, P. (ed.) MFCS 1980. LNCS, vol. 88, pp. 261-274. Springer, Heidelberg (1980)

11. Hermida, C., Jacobs, B.: Structural induction and coinduction in a fibrational setting. Information and Computation 145(2), 107-152 (1998)

12. Hesselink, W.H., Thijs, A.: Fixpoint semantics and simulation. TCS 238(1-2), 275$311(2000)$

13. Hughes, J., Jacobs, B.: Simulations in coalgebra. TCS 327(1-2), 71-108 (2004)

14. Kamae, T., Krengel, U., O'Brien, G.L.: Stochastic inequalities on partially ordered spaces. The Annals of Probability 5(6), 899-912 (1977)

15. Larsen, K.G., Skou, A.: Bisimulation through probabilistic testing. Information and Computation 94(1), 1-28 (1991)

16. Levy, P.B.: Boolean precongruences (2009) (journal submission)

17. Rutten, J.: Universal coalgebra: a theory of systems. TCS 249(1), 3-80 (2000)

18. Sokolova, A.: Coalgebraic analysis of probabilistic systems. Ph.D. thesis, Technische Universiteit Eindhoven (2005)

19. Staton, S.: Relating coalgebraic notions of bisimulation. In: Kurz, A., Lenisa, M., Tarlecki, A. (eds.) CALCO 2009. LNCS, vol. 5728, pp. 191-205. Springer, Heidelberg (2009)

20. Ulidowski, I.: Equivalences on observable processes. In: Scedrov, A. (ed.) Proceedings of the 7th Annual IEEE Symposium on Logic in Computer Science, pp. 148 161. IEEE Computer Society Press, Santa Cruz (June 1992)

21. de Vink, E.P., Rutten, J.J.M.M.: Bisimulation for probabilistic transition systems: a coalgebraic approach. Theoretical Computer Science 221 (1999)

22. Worrell, J.: Coinduction for recursive data types: partial orders, metric spaces and $\omega$-categories. ENTCS 33 (2000) 